\title{
ESCALA DE ATITUDE NA GRAVIDEZ: ADAPTAÇÃO PARA A CULTURA BRASILEIRA
}

\author{
Ana Márcia Spanó Nakano* \\ Marli Villela Mamede** \\ De Anne K. H. Messias***
}

\begin{abstract}
RESUMO: Este estudo objetivou realizar uma adaptação da escala Pregnancy Attitude Index (PAI) para a cultura brasileira. Tal instrumento constitui um recurso que a enfermeira obstétrica pode utilizar para conhecer as percepções das gestantes assistidas nos cursos de preparação psicoprofilática frente a experiência de parto, conduzindo dessa forma para uma assistência mais condizente com as reais expectativas da gestante. Como resultado desse processo, o instrumento mostrou-se com boa estimativa de confiabilidade ou consistência interna, bem como permitiu agrupar padrões de respostas emitidas pelas gestantes e identificar itens que necessitam de maior adequacidade. Acredita-se, no entanto, que o PAl está em condição de ser utilizado na cultura brasileira.
\end{abstract}

\begin{abstract}
Pregnancy Attitude Index-Adaptation for Brazilian Culture: The purpose of this study was to adapt the Pregnancy Attitude Index (PAI) for use in Brazilian culture. This instrument is a tool which obstetrical nurses can use to measure the perceptions of pregnant women who are enrolled in childbirth preparation classes, in order to better cater to the expectations of each woman. As a result of the process, the instrument was shown to have a good degree of reliabity and internal consistency. It was also possible to group the response patterns of the women tested, and identify items of the instrument which need to be adjusted. It appears, however, that the PAI is suitable for use in Brazilian culture.
\end{abstract}

\section{INTRODUÇÃO}

O surgimento dos métodos de preparação psicoprofilática para o parto nos anos 40 , veio em resposta ao pressuposto de que a sensação de dor é em parte socialmente aprendida e de caráter emocional. Atualmente, tais métodos tem sido utilizados mais como um artificio complementar da assistência obstétrica, do que como um recurso para um parto sem dor.

Entretanto, observa-se que a busca por esta preparação psicoprofilática para o parto não é uma constante entre as gestantes, e que, entre aquelas que participam de tais programas, o desempenho do tra- balho de parto e parto depende da percepção que elas têm sobre a relevância de seu comportamento na determinação do parto.

O locus de controle é uma variável da personalidade que permite explicar a percepção das pessoas frente a um dado evento. Derivada da Teoria do Aprendizado Social de ROTTER ${ }^{(5)}$. constitui uma ferramenta que permite interpretar as observações feitas pelas pessoas em resposta a questões sobre causalidade $^{(2)}$

Dessa forma, de acordo com ROTTER ${ }^{(5)}$. podese considerar duas dimensões para o locus de controle: interno, quando o indivíduo percebe que suas

\footnotetext{
* $\quad$ Professora Assistente do Departamento de Enfermagem Materno-Infantil e Saúde Pública da Escola de Enfermagem de Ribeirão Preto-USP.

** Professora associada do Departamento de Enfermagem Matemo-Infantil e Saúde Pública da Escola de Enfermagem de Ribeirão Preto - USP.

*** Professora Assistente do Departamento de Enfermagem da Faculdade de Ciências Médicas da Universidade de Campinas (UNICAMP).
} 
próprias ações podem exercer influência na situação vivenciada por ele, e externo, quando a percepção de controle se apresenta extra à pessoa, ou seja, o seu comportamento ou ação, não são determinantes na situação. $\mathrm{O}$ controle pertence a algum elemento fora de si próprio.

LEVENSON $^{(3)}$ elaborou uma escala de mensuração de Locus de Controle, adaptada da teoria social de Rotter, isolando três componentes distintos: controle interno (da própria pessoa), outros poderosos (outras pessoas), ao acaso (soite ou azar). Os dois últimos estão direcionados para a dimensão externa ${ }^{(4)}$.

O conceito de locus de controle vem sendo empregado por alguns estudiosos na área de psicoprofilaxia obstétrica. WILLMUTH(7) investigou a associação entre a satisfação com o parto preparado e o locus de controle. O'CONNELL ${ }^{(4)}$ utilizou a variável locus de controle para verificar a expectativa das gestantes frente ao parto, e SCHROEDER ${ }^{(6)}$ buscou avaliar a congruência entre as expectativas de controle frente ao parto (escala de locus de controle pré-parto) e o controle vivenciado durante o parto (escala de locus de controle pós-parto).

Considerando a existência de duas escalas de locus de controle, específicas para a situação em estudo, optouse pelo instrumento de O'CONNELL ${ }^{(4)}$, por ser uma escala aplicada á gestante na determinação da tipologia dos sujeitos, quanto ao locus de controle antes da ocorrência do parto, momento em que ocorrem as ações preparatórias para o mesmo.

A escala construída por O'CONNELL ${ }^{(4)}$, é de nominada Pregnancy Altitude Index - PAI. Busca verificar o locus de controle das gestantes em relação à experiência de parto, mensurar o quanto há de expectativa de controle pessoal (interno) e o quanto há de expectativa de controle extra á sua pessoa (externo).

A importância do conhecimento da localização, no contínuo interno-externo, dessa variável da personalidade da gestante para a prática de enfermeira obstétrica, está no planejamento de assistência, na preparação psicoprofilática, que venham atender ás reais expectativas da mulher grávida.

Dessa forma, cada gestante é considerada na sua individualidade, ou seja, uma mulher com locus de controle muito interno deve ser preparada para a possibilidade de intervenção no momento do parto, que está fora de suas capacidades de controle (como o caso de sofrimento fetal), o que diminuiria a sensação de culpa, trauma e fracasso da mu- lher, caso isso venha ocorrer. Na gestante com locus de controle muito externo, as ações devem ser no sentido de conduzi-la a uma maior participação, já durante a fase de preparação psicoprofilática.

Este trabalho tem como finalidade adaptar para a cultura brasileira um instrumento de medida da percepção da gestante em relação ao parto - "Pregnancy Attitude Index"(PAI), construído para â cultura norteamericana.

Portanto, são os objetivos desse trabalho:

1. Testar a confiabilidade do instrumento "Pregnancy Attitude Index"(PAI), com versão para a língua portuguesa.

2. Verificar se o "Pregnancy Attitude Index"(PAI), culturalmente apropriado para os Estados Unidos, pode ser utilizado para determinar as expectativas das mulheres brasileiras grávidas, em relação à experiência de parto.

3. Propor adaptações do "Pregnancy Attitude Index"(PAI) para a cultura brasileira.

\section{METODOLOGIA}

Instrumento de Medida: - Pregnancy Altitude Index (PAI), é uma escala construída por O'CONNELL ${ }^{(4)}$ baseada na de LEVENSON3-Levenson Tridimensional Locus of Control Scale.

O PAI contém 24 itens cujo conteúdo versa sobre as expectativas de controle da gestante em relação ao parto, associado com o aprendizado em programas de preparação psicoprofilática.

Os 24 itens contêm afirmativas destinadas a verificar as 3 dimensões do locus de controle, sendo 8 para controle interno, 8 para controle de outras poderosas e 8 para controle do acaso.

Em cada item é pedido â gestante indicar o nível de concordância ou discordância, variando de um contínuo de 6 (seis) pontos de tipo escala Likert: concordo totalmente: concordo; incerto, mas provavelmente concordo; incerto, mas provavelmente discordo; discordo e discordo totalmente.

Para análise do locus de controle deve-se somar os pontos obtidos para cada item. De acordo com O'CONNELL ${ }^{(4)}$ os escores variam de +3 a -3 , desde "concordo totalmente" até "discordo totalmente".

O cálculo dos itens de cada dimensão do locus é feito separadamente, e ao resultado deve-se somar +24 pontos, podendo variar de 0 a 48 pontos. Desta 
forma, cada sujeito recebe três escores, que indicam se o locus de controle é alto ou baixo nas três dimensões: controle interno, controle de outros poderosos e do acaso.

\subsection{Translação do instrumento Pregnancy Attitude Index (PAI)}

a) $\mathrm{O}$ instrumento foi traduzido para o português por três tradutores independentes, residentes no Brasil.

b) As três versões em português foram comparadas, analisadas e então elaborada uma nova versão por outros dois tradutores (dois dos pesquisadores). Nessa etapa realizou-se a validação aparente do conteúdo. A única modificação feita em relação ao conteúdo foi em relação ao termo trabalho de parto, permanecendo apenas o termo parto, pois em nossa cultura, especialmente no meio leigo, não é comum a diferenciação das etapas do processo de parturição, sendo a palavra parto utilizada para representar todo o processo.

c) Essa nova versão em português foi dada a outro tradutor, residente no Brasil, que domina os dois idiomas, inglês e português, para que o mesmo fizesse a versão de português para o inglês (back translation).

d) A nova versão em inglês foi encaminhada a O'CONNELL, autora da escala PAI, para comparação e análise do conteúdo na escala da back translation. Após essa analise a autora da escala enviou-nos algumas sugestões e a aprovação do conteúdo.

e) Após as correções, a escala em português foi confeccionada, bem como acrescentada de uma folha de instrução para preenchimento do instrumento (em anexo).

Em nenhum destes passos foi encontrada dificuldade na versão.

O passo seguinte foi aplicar o instrumento para testar sua confiabilidade.

\subsection{Amostragem e coleta de dados}

O estudo foi realizado com gestantes participantes do curso de preparação psicoprofilática para o parto, promovido por um hospital de médio porte do interior paulista, que atende a uma clientela particular e conveniada.

A amostra consistiu de 19 gestantes pertencentes a três tipos de preparação psicoprofilática para o parto, todas brasileiras, casadas, sendo que 15 são primigestas e 4 multíparas.

Os critérios de inclusão era ser gestante e participante dos cursos de preparação psicoprofilática para o parto.

A aplicação do instrumento foi realizada durante o primeiro dia do curso, coletivamente. Foram lidas as instruções para o preenchimento do mesmo e esclarecidas as possíveis dúvidas apresentadas.

\subsection{Análise dos dados}

Foi realizado o teste Alpha de Cronbach para verificar a confiabilidade e consistência interna do PAI. Foram também calculadas as médias, variâncias, freqüências das respostas de cada item do instrumento.

\section{RESULTADOS E DISCUSSÃO}

A distribuição da freqüência, média e variância das respostas aos 24 itens da escala PAI, segundo as dimensões do locus de controle estão apresentadas na Tabela 1.

Ao teste Alpha de Cronbach, usado para testar a confiabilidade e consistência interna das respostas aos 24 itens do instrumento PAI, este mostrou-se confiável diante do valor 0,74 .

Considerando os itens nas 3 dimensões do locus de controle, observamos que houve uma dispersão das respostas no contínuo concordância-discordância, que podem ser constatados pela inexistência de valor zero na variância.

No entanto, observa-se que no item 18. Eu posso determinar muito a evolução do meu parto, houve uma variância extremamente baixa $(0,23)$, o que nos leva a questionar se esse resultado significa que realmente o grupo concorda com esta afirmação ou se é devido à não compreensão do conteúdo.

Ao lado disso, acreditamos que a forma como o instrumento foi aplicado, coletivamente, limitou os esclarecimentos apenas àqueles solicitados. Se usada a entrevista tipo face-face, haveria melhor oportunidade para detectar itens não compreensíveis ou melhor observar aqueles onde a variabilidade é pequena.

Diante dessas considerações, acreditamos que há necessidade de rever a adequacidade dos itens 4,5 e 18 , que obtiveram menor variância.

$\mathrm{Na}$ análise das três dimensões do locus de controle quanto à freqüência e médias das respostas, há 


\section{Tabela 1}

Distribuição das freqüências, médias e variâncias das respostas por ítens nas três dimensões do locus de controle.

\begin{tabular}{|c|c|c|c|c|c|c|c|c|c|}
\hline Dimensão & $\begin{array}{cc}\text { Concordo } \\
\text { Itens } & \text { totalmente }\end{array}$ & Concordo & $\begin{array}{l}\text { Incerto, mas } \\
\text { provavelmen- } \\
\text { te concordo }\end{array}$ & $\begin{array}{l}\text { Incerta mas } \\
\text { provontrete } \\
\text { decordo }\end{array}$ & Discordo & $\begin{array}{l}\text { Discordo } \\
\text { totalmente }\end{array}$ & $\bar{x}$ & Var & \\
\hline \multirow{3}{*}{$\begin{array}{l}\stackrel{\circ}{\subseteq} \\
\stackrel{ \pm}{\subseteq}\end{array}$} & $10 \%$ & $47 \%$ & $31 \%$ & $0 \%$ & $21 \%$ & $0 \%$ & 2,94 & 1,31 & \\
\hline & $416 \%$ & $58 \%$ & $26 \%$ & - & - & - & 2,10 & 0,40 & \\
\hline & $516 \%$ & $58 \%$ & $26 \%$ & - & - & - & 2,10 & 0,40 & \\
\hline \multirow{4}{*}{$\begin{array}{l}\frac{0}{0} \\
\text { 돈 } \\
\text { O }\end{array}$} & $9-$ & $16 \%$ & $47 \%$ & - & $31 \%$ & $5 \%$ & 3,63 & 1,49 & \\
\hline & 18 & $5 \%$ & $74 \%$ & $21 \%$ & - & - & - & 2,15 & 0,23 \\
\hline & 19 & $21 \%$ & $58 \%$ & $16 \%$ & $5 \%$ & - & - & 2,05 & 0,57 \\
\hline & 21 & $56 \%$ & $42 \%$ & $31 \%$ & - & $25 \%$ & - & 2.89 & 1.46 \\
\hline \multirow{6}{*}{ 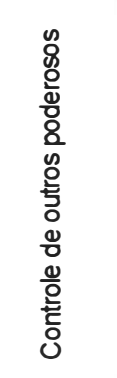 } & 23 & - & $16 \%$ & $37 \%$ & $16 \%$ & $26 \%$ & $5 \%$ & 3,68 & 1,37 \\
\hline & $3-$ & $11 \%$ & $5 \%$ & $11 \%$ & $42 \%$ & $31 \%$ & 4,78 & 1,53 & \\
\hline & & $37 \%$ & $42 \%$ & - & $21 \%$ & - & 3,05 & 1,20 & \\
\hline & 11 & - & $16 \%$ & $11 \%$ & $16 \%$ & $31 \%$ & $26 \%$ & 4,42 & 1,92 \\
\hline & 13 & - & $26 \%$ & $47 \%$ & - & $26 \%$ & - & 3,26 & 1,24 \\
\hline & 15 & - & $5 \%$ & - & $5 \%$ & $63 \%$ & $26 \%$ & 5,05 & 0,78 \\
\hline \multirow{3}{*}{ 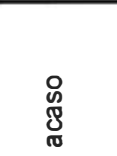 } & 17 & - & $21 \%$ & $37 \%$ & $16 \%$ & $26 \%$ & - & 3,47 & 1,19 \\
\hline & 20 & $5 \%$ & $21 \%$ & $21 \%$ & $11 \%$ & $42 \%$ & - & 3,63 & 1,81 \\
\hline & 22 & $5 \%$ & $21 \%$ & $42 \%$ & $11 \%$ & $16 \%$ & - & 3,10 & 1,14 \\
\hline \multirow{3}{*}{$\begin{array}{l}\text { 응 } \\
\text { 잉 } \\
\text { 등 }\end{array}$} & $2-$ & - & $26 \%$ & $5 \%$ & $58 \%$ & $11 \%$ & 4,52 & 0,98 & \\
\hline & $6-$ & $16 \%$ & - & $26 \%$ & $31 \%$ & $26 \%$ & 4,52 & 1,72 & \\
\hline & $75 \%$ & $21 \%$ & $47 \%$ & ‘5\% & $21 \%$ & - & 3,15 & 1,29 & \\
\hline
\end{tabular}

uma tendência à concordância para as afirmações dos itens destinados ao controle interno. A maior variância para as respostas dos itens do locus cie controle outros poderosos e ao acaso, reflete na média e freqüência das respostas a estes itens, prevalecendo uma mobilidade para incertezas e discordâncias.

Os resultados possibilitaram a identificação de locus de controle nas três dimensões do grupo como um todo, a saber: dimensão interna $=32$ pontos, outros poderosos $=22$ pontos e ao acaso $=20$ pontos, significando na escala de $0-48$, que o grupo estudado tem a percepção de que suas próprias ações são importantes na determinação da experiência de parto (controle interno).

As dimensões do locus de controle externo (outros poderosos e ao acaso) obtiveram um escore abaixo da média da escala $0-48$, o que denota que o grupo não percebe como de grande influência os fatores externos à pessoa na determinação da experiência de parto.

\section{CONSIDERAÇÕES FINAIS}

A escala Pregnancy Attitude Index (PAI) aplicada a 19 gestantes participantes dos programas de preparação psicoprofilática para o parto obteve uma boa estimativa de confiabilidade ou consistência interna $(0,74)$, segundo o coeficiente Alpha de Cronbach.

A análise dos resultados permitiu agrupar padrões de respostas emitidas pelas gestantes, bem como foi possível identificar alguns itens que não se obteve padrão nas respostas.

Diante dos resultados encontrados, acredita-se que o PAI está em condições de ser utilizado na cultura brasileira. 


\section{REFERÊNCIAS BIBLIOGRÁFICAS}

1. CHAIPHIBALSARISDI, P. Process of translation of measurement, self care responses questionnaire (SCRD). University of Illinois at Chicago. College of Nursing, 1987. (unpublished).

2. DELA COLETA, J.A. A Escala de locus de controle InternoExterno de Rotter: um estudo explanatório. Arq. Bras. Psicol., v.31, n.4, p.167-81. 1979

3. LEVENSON, H. Multidimensional locus of control in psychiatrie patients. Journal of Consulting and Clinical Psychology, v.41, n.3, p.397-404, 1973

4. O'CONNELL, M.L. Locus of Control Specific of Pregnancy. JOGN Nursing, v.12, n.3, p.161-4, 1983
5. ROTTER, J.B. Generalized expectancies for internal versus external controle of reinforcement. Psychological Monographis. General and Applied, Washington. 80. (Whole no.609), 1966.

6. SCHROEDER, M.A. Development and testing of scale to measure locus of control prior and following childbirth Maternal Child Nursing Journal, v. 14. n.2. p.111-21, 1985.

7. WILLMUTH, R. et al. Satisfaction with prepared childbirth and locus of controle. JOGN Nursing, v.7, n.3, p.33-7. 1978. 


\section{ANEXO \\ INSTRUÇÃO}

Vamos fazer umas perguntas a você sobre o que as pessoas sentem e pensam da gravidez e do parto, para melhor prepará-la por ocasião do nascimento de seu filho. A melhor resposta para cada uma das afirmações é a sua opinião pessoal Tentamos abordar muitos pontos de vista diferentes. Você poderá concordar com algumas das afirmações, discordar de outras, e ter dúvidas em outras. É importante você saber que qualquer que seja sua resposta, muitas pessoas sentem e pensam como você. Certo?

Em cada afirmação você encontrará as letras: A, B, C, D. E e F. Veja em qual letra está a resposta que você mais concorda e coloque um $\mathrm{X}$.

\section{POR EXEMPLO:}

1) O fato de eu ter, ou não, uma boa experiência no parto, depende principalmente de como eu utilizo as técnicas que aprendi nas aulas.
A) Concordo totalmente
B) Concordo
C) Incerta, mas provavelmente concordo

D) Incerta, mas provavelmente discordo

E) Discordo

F) Discordo totalmente

Você Compreendeu? Ótimo! Vamos começar?

01) O fato de eu ter, ou não, uma boa experiência no parto, depende principalmente de como eu utilizo as técnicas que aprendi nas aulas.

02) O que acontecer durante a minha gravidez dependerá em grande parte do acaso.

03) Acho que os acontecimentos durante a minha gravidez serão determinados, principalmente, por outras pessoas que exerçam controle sobre mim.

04) Tenho mais chances de ter um bom parto se eu conseguir fazer bem o que aprendi nas aulas.

05) É mais provável que eu tenha o tipo de experiência que eu quero, se planejar minha conduta no parto, assistindo às aulas.

06) Não tenho como proteger minha gravidez contra os azares da vida.

07) Se essa gravidez tiver os resultados que espero, isso será porque eu tive sorte.

08) Apesar da minha capacidade de fazer o que aprendi nas aulas, o tipo de experiência que terei no parto será controlado principalmente pelo meu médico.

09) O médico gostará mais de mim se eu conseguir manter o controle durante o parto.

10) Acredito que se tiver de acontecer alguma coisa para mim e ao meu futuro bebê, acontecerá de qualquer jeito. 
11) As normas do hospital serão as maiores responsáveis por eu ter, ou não, o tipo de experiência no parto que eu quero.

12) O fato de eu ter, ou não, a experiência que eu quero, depende sobretudo da sorte.

13) A maioria das pessoas tem pouca influência sobre o que querem durante o parto, se isso contrariar as normas do hospital.

14) Não faz muito sentido se preparar para o parto, já que muitas coisas dependem da sorte ou do azar.

15) Acho que se eu agradar o meu médico, ele prestará mais atenção em mim.

16) O que acontece durante a gravidez é determinado principalmente pelo fato de você ter sorte de uma gravidez saudável.

17) Quando o médico gosta de uma paciente, esta tende a receber melhores cuidados.

18) Eu posso determinar em grande parte o andamento do meu parto.

19) Conhecendo os meus direitos, serei capaz de proteger meus interesses durante o parto.

20) O fato de eu ter, ou não, uma complicação depende de como o meu médico cuida de minha gravidez.

21) Se eu tiver o tipo de parto que eu quero, será porque me esforcei para isso.

22) Se eu seguir cuidadosamente as instruções do meu médico, terei o tipo de parto que eu quero.

23) Meu parto será determinado pelas minhas próprias ações.

24) Que eu tenha um bebê saudável ou não dependerá sobretudo do destino. 